\title{
El léxico de la isla del Hierro*
}

Al estudiar la lengua de las islas, más de una vez se ha destacado la personalidad del Hierro. El ALEICan nos permite un acercamiento al léxico herreño y la comparación con el del resto del archipiélago ${ }^{1}$.

Lo primero que se advierte al consultar el mapa 3 es que a la pregunta «¿Qué hablan aquí?», El Hierro contesta unánime berreño, mientras que en las demás islas, salvo casos aisladoss de gomero (Go 4), canario (Gc 40), majorero (Fv. 20, 30) y habla de mago (Tf. 6), lo habitual es español o castellano ${ }^{2}$. Esta conciencia de su propia habla quizá se deba, en parte, a que la fonética del Hierro, en el panorama general de las islas, resulta conservadora: lleísmo frente a la expansión desde las ciudades del yeismo, $[\stackrel{\mathfrak{t}}{\mathrm{s}}]$ con La Palma frente a la $[\hat{\mathrm{y}}]$ de las demás, conservación de -d- intervocálica, no confusión de $\mathrm{r}$ y 1 implosivas y sólo en ocasiones aspiración de $-s$ final ${ }^{3}$.

Pero esta singularidad del Hierro también se aprecia en su léxico. Son dos sus características principales: la independencia, que determina su ori-

* Este trabajo se presentó en el III Simposio Internacional de Lengua Española (Las Palmas, 1984). Hay información posterior en A. LloRente MALDONADO DE Guevara, «El léxico del tomo I del "Atlas Lingüístico y Etnográfico de las Islas Canarias"» (Cáceres: Anejos del Anuario de Estudios Filológicos, 1987) y en el libro póstumo de J. Pérez Vidal, Los portugueses en Canarias. Portuguesismos (Las Palmas: Eds. del Cabildo Insular de Gran Canaria, 1991).

Utilizaré los dos primeros tomos del ALEICan, ya que el tercero está dedicado al mar: M. Alvar, Atlas lingüístico y Etnográfico de las Islas Canarias (Las Palmas: Ediciones del Excmo. Cabildo Insular, t. I, 1975; t. II, 1976).

2 Ya J. Fernández Sevilla lo señaló en «Andalucía y Canarias: relaciones léxicas», I Simposio Internacional de Lengua Española (1978) (Las Palmas: Eds. del Excmo. Cabildo Insular, 1981), p. 110. Las islas marginales prefieren castellano a español, mientras que los términos se invierten en las grandes islas. M. Alvar en «Actitud del hablante y sociolingüística», Teoría lingüística de las regiones (Barcelona: Planeta, 1975), pp. 93114, consideró expresamente la discrepancia del Hierro en este sentido (p. 100).

3 M. Alvar, «La articulación de la ' $s$ ' herreña», Mélanges offerts à M. Georges Straka (Lyon-Strasbourg, 1970), I, pp. 105-114. Allí, p. 109, afirmaba que «en la isla del Hierro hay tendencia a conservar la $-s$ en posición final absoluta», sin que parezcan existir razones socioculturales para ello y que la $s$ herreña no es, como se escribió, distinta de la general en Canarias, sino «predorsoalveolar, muy dentalizada». 
ginalidad, y la uniformidad. Ya en un anterior Simposio, al estudiar la Originalidad interna en el léxico canario, Manuel Alvar señalaba cómo, en El Hierro, de 96 mapas, «nada menos que cuarenta palabras (un 41,66\%) son discrepantes con respecto a las demás islas», resultando afectados todos los campos ideológicos, especialmente el ganadero ${ }^{4}$. En cuanto a su uniformidad, de los 789 mapas que forman los dos primeros tomos del ALEICan, casi 300 presentan una sola voz para un objeto o concepto en toda la isla. Uniformidad léxica propiciada, sin duda, por su reducida extensión, pero también por su concreción lingüística.

El léxico del Hierro hace, como es natural, referencia al medio: hay blancos significativos en los mapas del ALEICan. No se pudo recoger allí lo referente al cultivo del plátano, ni el léxico de las palmeras. Tampoco las palabras relacionadas con los dromedarios, el carro o el yugo de vacas. La realidad condiciona el lenguaje, pero el cultivo de la vid, los cereales, las higueras y la ganadería proporcionan materiales ricos que permiten su caracterización. El Hierro posee un léxico fundamentalmente castellano, pero sobre él se asientan muchos elementos occidentales, sobre todo portugueses, además de voces prehispánicas, americanas y marineras.

\section{PORTUGUESISMOS}

La influencia de la cultura tradicional portuguesa en las islas Canarias ha sido objeto de muchos estudios s. Fruto de esta influencia es la incorporación de portuguesismos a las hablas del Archipiélago. El Hierro suele participar de los generales: andoriña 'golondrina', arco de la vieja 'arco iris', bagaso 'orujo', balango 'avena loca', baña 'manteca', bica / biquera 'piquera del lagar', bicariño 'higo pequeño y oscuro', bosta 'boñiga', bubango 'calabacín insular' (si se acepta un origen port. bubão), coruja 'lechuza', corcovado 'jorobado', enganso 'escobajo', escaida 'gajo del racimo', escarro 'gargajo', fechillo

4 Actas del I Simposio Internacional de Lengua Española, p. 254. Allí El Hierro encabeza la escala de discrepancia con un 41,64\%,seguido muy de lejos por La Gomera con un 11,45\%, Gran Canaria 10,41, La Palma 9,37, Lanzarote 6,25, Fuerteventura 6,26 y Tenerife 4,16.

5 J. Pérez Vidal, «Portuguesismos en el español de Canarias», Museo Canario, 9 (1944); ID., «Influencia portuguesa en la cultura tradicional marinera de Canarias», separata de las Actas do Congresso Internacional de Etnografia (1963) (Lisboa, 1965); ID., «Esbozo para un estudio de la influencia portuguesa en la cultura tradicional canaria», Homenaje a E. Serra Rafols (Universidad de La Laguna: 1970), I, pp. 371-390. Para la identificación de los posibles portuguesismos utilizo el Novo Diccionário da Língua Portuguesa de Candido Figueiredo (Lisboa: Livraria Clássica Editora, 1913), II. 
'pasador de hierro', ferrojo 'cerrojo', fogar 'hogar', fonil 'embudo', furo 'agujero por donde se echa el grano' (Hi 1), furos 'clavijero' (Hi 1, 2), fol 'odre en el que se mece la mantequilla', legumen 'legumbre', millo 'maíz', maravalla 'viruta', pargana 'argaya', petudo 'jorobado', picar 'guiñar los ojos', remoler 'rumiar', serrote 'serrucho', sorribar 'roturar'/'descuajar', talla 'vasija para recoger el agua filtrada', tarsuelo 'orzuelo', traza 'carcoma', urrar 'mugir', verode/verol 'planta' 6 .

También hay en la isla una serie de palabras a las que se les puede suponer un origen portugués, pero que el ALEA documenta con mayor o menor extensión en la Andalucía occidental ': abanador 'soplillo' y abanar 'soplar' (ALEA III, 726: en Huelva con entradas en Sevilla), alpendre 'cobertizo' (ALEA II, 464: alpende 'establo de vacas' en H 204 y II, 679: alpende 'cobertizo' en H 101, 501, 204, 602, 603 y Se 301), margullir 'chapuzar' (ALEA V, 1446: margullarse en $\mathrm{H} 303$, dar un margullón en $\mathrm{H} 101$ y 504, mergullón en $\mathrm{H} 102$, morgullirse en $\mathrm{H} 200$ ) y roncollo 'rencoso' (que aparece en toda Huelva y entra en Sevilla como rencollo, para más al E. tener tratamiento castellano: rencojo en Sevilla, Córdoba y Jaén: ALEA II, 509).

Otros casos como los de camellón 'caballón', amolador 'afilador', maestra 'abeja reina', hacer un penino 'hacer el pino', cuadril 'cadera', lamber/lambiar 'lamer' o verija 'ingle', a pesar de su coincidencia con formas portuguesas, por su mayor extensión en Andalucía occidental, sólo pueden calificarse de occidentalismos.

Pero El Hierro se caracteriza por participar del portuguesismo marcado de las islas occidentales, coincidiendo a veces con La Palma, otras con Gomera y Tenerife. Un ejemplo lo tenemos en la voz marco que corresponde al castellano 'mojón'. Marco, general en El Hierro, sólo reaparece en dos puntos de La Palma, donde alterna con mojones, y en uno de Gran Canaria ${ }^{8}$. El ALEA ratifica el origen de la voz al documentarla en los puntos fronterizos

6 Si admitimos una relación con el berol o pepino-do-mar, planta submarina de la que habla Figueiredo. Ver M. Alvar, El español bablado en Tenerife (Madrid: CSIC, 1959), p. 251, y María Inmaculada CoRRALES, «Contribución al estudio del léxico canario», I Simposio..., pp. 184-186.

7 De hecho, algunos de los portuguesismos citados arriba se puede decir que lo son por su aparición limitada a los puntos fronterizos del ALEA. Por ejemplo, balango avena loca' es balanco en H 100 (ALEA I, 293), enganso 'escobajo' sólo en H 303, 501 y 504 (ALEA II, 454), serrote sólo en H 101 y acerrote en H 504 (ALEA IV, 936), tarzuelo es torzuelo en H 101 (ALEA V, 1204), traza sólo en H 504 (ALEA IV, 938) o urrar sólo en H 501 (ALEA II, 472).

8 I, 14. LP 10 y 30 y GC 20. 
de Huelva 9 . Figueiredo define marco como «Pedra oblonga, cona que se demarcam terrenos. Qualquer pedra, de situação natural e que se aproveita para sinal de limites territoriaes», lo que concuerda con el hecho de que en las islas occidentales todos se hagan con una piedra hincada ${ }^{10}$. Surco es segunda respuesta en Hi 2 y 10, pero la primera y general en toda la isla es rego, portuguesismo que en La Palma retrocede a un segundo lugar frente a surco en tres localidades y se señala como anticuado en otra ${ }^{11}$. También une El Hierro con La Palma la conservación de derivados del latín i n d e x como 'nidal'. El herreño nidés y los palmeños endé, lendé, nendé no son prehispánicos, como ya estableció M. Alvar ${ }^{12}$, mantienen el portugués êndez o êndes, «Ovo, que se colloca no lugar em que se deseja que uma gallinha ponha outros».

El mapa I, 267 '(higos) bergazotes' recoge en La Palma y El Hierro siempre cotio, respuesta bastante extendida en Tenerife donde alterna con coto y bregasote. Se trata de un lusismo no señalado antes, pues cotia es «Casta de figueira algarvía» y cotio «Variedade de figo branco e grande». Otro portuguesismo aislado esta vez en Hi 2, poscases 'pescuño' sólo tiene paralelo en el poscase de LP 10 y 20, todos procedentes del portugués pescaz, «Cunha com que se une o arado á rabiça» ${ }^{13}$.

En ocasiones El Hierro comparte sus portuguesismos sólo con Tenerife. Ocurre así con espirrar 'estornudar' en los puntos interiores de la isla: se trata de un caso de conservadurismo, ya que los puntos marineros 2 y 3 contestan estornudar, aunque $\mathrm{Hi} 2$ da espirrar como segunda respuesta y, fuera del Hierro, sólo se registra en Tf $5^{14}$. También ingua 'hernia' $<$ del portugués ingua 'id.' es propio del interior del Hierro y se localiza en distintos puntos de Tenerife. El portuguesismo, como ocurre en otros muchos

9 ALEA I, 13. H 102, 204, 302, 301, 303. En H 101 alterna con mojón y en $\mathrm{H}$ 100 marco es el mojón blanqueado.

10 ALEICan I, 15 mapa etnográfico.

11 I, 30. Rego LP 1, 20, 3 y LP 10. Triunfa, como ocurre a menudo, un sentido secundario de rêgo que en primer lugar es «Sulco, natural ou artificial, que conduz água ou é própio para isso» y sólo después «Sulco, feito pelo arado». Curiosamente en Hi 2, donde conviven surco y rego, los sentidos se restringen y se especializan, resultando surco 'surco donde se siembra el maíz' y rego 'surco donde se siembra cebada o centeno'. El informante de Hi 4 sabía también que surco era el nombre de otros sitios. Ver también J. FERNÁNDEZ SEVILla, art. cit., p. 76.

12 II, 417. Ver M. AlVAR, Originalidad, $\$ 1.60$. Las formas palmeñas con aglutinación de artículo y todas con cambio acentual que también presenta el único caso registrado en el ALEA (II, 160), andé, en la frontera de Huelva (H 501).

$13 \mathrm{I}, 115$.

14 II, 913: espirrar Hi 1, 4, 10. Del portugués espirrar «Expellir, lançar fora. Dar espirros». 
casos, se repliega frente a voces castellanas: en $\mathrm{Hi} 3$ «está quebrado», en Hi 2 bernia, pero allí ingua es 'bulto que sale en alguna parte del cuerpo cuando tenemos una llaga o herida en otro sitio', aunque debió tener mayor difusión ya que en LP 3 y Lz 3 y 10 significa 'ingle' ${ }^{15}$.

La 'vasija para ordeñar' es en $\mathrm{Hi}$ 1, 2, 4 tarro frente a jarro en $\mathrm{Hi} 10$. En este caso habría que suponer una relación palabra-cosa ya que tarro es 'vasija de barro en la que se ordeña a las cabras y ovejas' (Hi 1), mientras que jarro es 'vasija de metal para ordeñar a las vacas'. El tarro herreño es, pues, el portugués «Vaso em que se recolhe o leite, quando êste se ordenha», por más que la existencia de la voz en español con un significado muy cercano la convierta en uno de esos portuguesismos encubiertos o solapados de los que habló Julio Fernández-Sevilla ${ }^{16}$. También deben ser lusismos de las islas occidentales mollo 'gavilla de centeno ya amarrada' ${ }^{17}$, bago 'grano de uva' ${ }^{18}$ y su derivado desbagar, y quizá masapés 'arcilla' ${ }^{19}$.

Los portuguesismos han retrocedido en las demás islas y también lo hacen en El Hierro. Las voces españolas los sustituyen en los puntos marineros: p. e. ferrojo (m. 549) del port. ferrolbo se conserva en el interior, mientras que cerrojo es respuesta de $\mathrm{Hi} 2$ y 3; lo mismo que el lusismo bagaso en

15 II, 496. Ingua Hi 1, 4, 10 y Tf 5, 6, 31, 41, 50. Caso similar al de Hi 2 es el de Tf 5, donde 'hernia' es rotura, pero ingua 'el bulto que sale al darse un golpe'. Ingua 'ingle' en ALEICan II, 495. Pero el primer sentido de la voz es 'hernia': Figueiredo la define como: «Engurgitamento de glândula lymphatica na virilha ou no pescoço ou na axilla. Bubão na virilha».

16 Art. cit., p. 103. Quizá entre estos últimos se cuenten rabiza (m. 114) nombre de la 'esteva' en El Hierro y La Palma y que en otras islas alterna con mancera, ya que en el ALEA se documenta como 'tralla', y aquí coincide con el port. rabiça "Rabo do arado", o sobar 'heñir' (m. 180) en Hi 1, 2, 10 y con gran difusión, que podría ser especialización del castellano sobar, pero que se ajusta al port. sovar «Amassar. Bater a massa».

17 I, 54. En Hi 10 y también en La Palma y Tenerife. Del portugués molbo «Feixe. Mão-cheia. Paveia».

18 I, 140. También en La Palma, Tenerife y Gomera. Aunque puede tratarse de un occidentalismo, su localización en Andalucía en H 100, 101, 200, 201 (ALEA I, 198) inclina hacia el portugués bago "Qualquer pequeno fruto redondo e carnudo, semelhante ao da uva».

19 II, 773. Hi 1, 2, 4, 10. En Tenerife es el nombre de la 'greda' (II, 774). Ver el comentario de M. Alvar, Originalidad, $\$ 1.96$. Más generales habrían sido mellares, nombre de los 'marranos de la prensa' en Hi 1 (I, 155) como segunda respuesta, con alguna localización también aislada en Tenerife, Gomera y Lanzarote, probablemente del portugués malhal «Travéssa de madeira, que assenta no pé da uva, dentro dos lagares, $e$ sôbre a qual carrega a vara do lagar», aunque habría que considerar su relación con mallal o mayal 'cerdo padre', y pedrero 'albañil', también en $\mathrm{Hi} 1$ y $\mathrm{Lz} \mathrm{1,2}$ y 3, que coincide con el port. pedreiro (II, 630). Pedrero, maestro pedrero en $\mathrm{Lz} 1,2$ y 3 . 
los puntos interiores, mientras Hi 2 prefiere orujo ${ }^{20}$. De cualquier forma, por su marginalidad y tendencia manifiesta a la uniformidad, El Hierro se ha convertido en «reserva» de portuguesismos que en otras islas han ido perdiendo su lucha con la lengua oficial. Algunos incluso presentan una fonética más próxima a su origen, como el portugués escádea 'gajo del racimo', que es escadia, escaida y escaiga en El Hierro, mientras que en La Palma, Gomera, Tenerife, Gran Canaria y Lanzarote, cuando se conserva, suele pasar a escada y de ahí, con mucha frecuencia, a escala y cala ${ }^{21}$. Algo semejante se observa en el mapa 359 'mugir' que en El Hierro es exclusivamente urrar, del portugués urrar «Dar urros; rugir» y que, en las demás islas, lo habitual es que se deforme en urriar, urrir, oral, burrar, burriar, aburriar, murriar o murrar ${ }^{22}$.

En otras ocasiones, el lusismo herreño tiene un sentido más especializado que el de otras islas: p.e. talla, que suele designar la 'tinaja', en la isla es 'la vasija para recoger el agua filtrada' ${ }^{23}$. Baña, del portugués banha «Gordura de animaes», llaman en todas las islas a la manteca del cerdo, pero en El Hierro bañas es 'hoja de tocino', lo mismo que baña o labbaña en puntos de La Palma y Gomera ${ }^{24}$. Un caso curioso es el de la coincidencia entre una designación secundaria del 'bocado de Adán o nuez' en El Hierro y Lanzarote. En las islas la voz general es manzana, presentando un proceso de creación léxica que acerca el canario a lenguas románicas con la misma

20 I, 156. También segunda respuesta en Hi 10. El ALEA I, 202 documenta gabazo en el sur de Huelva para 'escobajo'. Se ve claramente que el lusismo no es andaluz en Canarias no sólo por su forma, sino porque su significado está más próximo al portugués: "Resíduo dos frutos, que foram espremidos para se lhes extrahir o suco». I. LERNER, Arcaísmos en el español de América (Madrid: Ínsula, 1974) s.v., documenta bagazo en América como 'residuo de la caña de azúcar', que viene a coincidir con lo que, según el DRAE, se llama bagazo «en algunas partes».

21 I, 138. Escadia en Hi 10, escaida en Hi 1 y 2, escaiga en Hi 4.

22 El ALEA (II, 472) sólo lo recoge en un punto fronterizo de Huelva con Portugal (H 501: 「urrando, urrio).

23 II, 593. Aunque no hay tinajas, los herreños las distinguen y las llaman tinas o tinajas, no tallas en Hi 1 y 10. También el portuguesismo entullo, que con derrumbamiento recoge el significado de 'desprendimiento' en el Archipiélago, en El Hierro no aparece con ese sentido, para el que sólo conoce derrumbe (II, 798). De nuevo triunfa un segundo sentido del port. entulbo «Aquillo que enche ou entupe uma cavidade ou fôsso. Montão de caliça, proveniente de desmoronamento». En El Hierro su empleo está más cerca del primero, porque, en el mapa 206 Deshacer el horno, en Hi 4 entulla es 'pone tierra sobre el carbón', aunque en el mismo punto entullo significa 'gleba' (II, 772).

24 II, 462. Bañas (Hi 1, 2, 4), baña (LP 1), labbaña (LP 3 y Go 2). 
solución, desconocida en la Península ${ }^{25}$, pero en Hi 3 nudo de la garganta parece adaptar el portugués nó da garganta, como núo la garganta y nudillo (de)l garguero en Lanzarote, a menos que se trate de creaciones paralelas ${ }^{26}$.

Los portuguesismos que el ALEICan documenta sólo en El Hierro son pocos: entre ellos atillo 'vencejo' ${ }^{27}$, borrento 'vino turbio' ${ }^{28}$, churume 'zumo de la uva' ${ }^{29}$, carespas 'ramojo' y 'viruta' ${ }^{30}$, arrife 'ribazo' ${ }^{31}$, boba 'cidra' ${ }^{32}$, taño 'recipiente para guardar el trigo' ${ }^{33}$ y espicha 'huso de hilar' ${ }^{34}$.

Sorprende, en cambio, la ausencia de algunos lusismos que, por su extensión en Canarias, cabría esperar allí: el port. gomo 'gajo de la naranja' de Tenerife, en la isla se llama ruẹda, caso de creación propia sin antece-

25 A. Llorente Maldonado, «Comentario de algunos aspectos del léxico del tomo II del ALEICan», I Simposio Internacional de Lengua Española, p. 196.

26 II, 490. $\mathrm{Lz} 4$ y 30 respectivamente. De todas formas, Hi 3 es punto marinero y la voz puede ser importada.

27 Ver Alvar, Originalidad, \$ 1.5.

28 I, 162, documentado sólo en Hi 2. También Hi 2 da canento para 'canoso' (II, 482).

29 I, 229. Churume, anticuado en Hi 4, y surume (Hi 10). El DRAE da churumo 'jugo o sustancia', y F. Guerra, Contribución al léxico popular de Gran Canaria (Madrid, 1965), s.v., churrume, con lo que el término ya no debe ser exclusivamente herreño, para «lo que, ablandado por el calor u otras causas, se derrite y escurre denso y pegajoso», remitiendo a Corominas que considera portuguesa la voz.

30 I, 241 'ramojo' en Hi 2; II, 635 'viruta' en Hi 3. Del portugués carepa «Caspa, aspereza cutânea. Lanugem de alguns frutos. Superficie de madeira, cortada com enxó», un nuevo ejemplo de que los portuguesismos suelen conservarse con uno de sus sentidos secundarios, no con su significación principal.

31 II, 776 en Hi 2 y 10. Rife en Go 2. Se ha visto en la voz un guanchismo: A. ZAMOra Vicente, Dialectología española (Madrid: Gredos, 1967), p. 374. Sin embargo, GUERRA, op. cit., lo recoge en Gran Canaria como «pegujalejo o hacendilla de tierras pedregosas y eriales, con las peores especies de monte bajo por toda vegetación», y parece concordar con el sentido que arrife tiene en portugués de las Azores: «Tênue camada de terreno, em que aparecem aqui e ali, cabeçotes de rocha subjacente». Aurélio BUARQue de Holanda Ferreira, Novo Dicionário da Lingua Portuguesa (Rio de Janeiro: Ed. Nova Fronteira, 1." ed., 5." impr., s.a.) da como primer significado «Arrife. s.m. Recife. Penhascos no mar e à flor da água», como forma paralela de arrecife.

32 I, 228 en Hi 2.

33 I, 88. En Hi 2 es 'recipiente con bálago de centeno (6-12 arrobas) para guardar el trigo en los alpendes'. Figueiredo clasifica tanbo como voz principal del Algarve: «Grande seirão, própio para conter cereaes». Álvarez DelGADO, «Ecero», p. 157, incluye taño entre las palabras prehispánicas.

34 II, 628. La voz fue estudiada por M. Alvar, Originalidad, $\ 1.85$. 
dentes peninsulares ${ }^{35}$. Tampoco es herreño en el ALEICan el portuguesismo tabefe 'requesón'; se emplea exclusivamente el castellanismo requesones ${ }^{36}$, y también una voz castellana panzón 'estómago del cerdo' opone El Hierro al lusismo generalizado en otras islas, payo ${ }^{37}$. Rueda, requesones y panzón son, además, términos exclusivos de la isla.

Cabría señalar algún otro ejemplo como el de las denominaciones de la 'alondra', cantor o capirote en El Hierro, cuando las demás islas se inclinan por calandra ${ }^{38}$, o el del mapa 286 donde mariposa en todos los puntos herreños se opone a algún borboleta en La Palma ${ }^{39}$. En alguna otra ocasión El Hierro vuelve a oponer una voz castellana a lusismos de otras islas: lavijero 'clavija' frente a chaveta ${ }^{40}$, bongo 'seta' frente a tortullo en bastantes puntos de La Palma ${ }^{41}$, pasador o tranquita frente al general taramela ${ }^{42}$.

Sus portuguesismos son, en resumen, los de las islas occidentales con alguno más propio y con la ausencia de otros que parecen vencidos por su equivalente castellano. Destaca su presencia en el campo de la agricultura, no así en el ganadero, y suelen triunfar sus sentidos secundarios - como en

35 I, 254. De nuevo un lusismo insular parece conservar un segundo sentido, pues Figueiredo da en primer lugar «Rebento dos vegetaes, que se transforma em ramo ou folha», y sólo en segundo lugar "Cada uma das divisões naturaes de certos frutos, como a laranja». Pero ese sentido tiene también en los puntos fronterizos de Huelva (ALEA II, 352: $\mathrm{H} 303,500,501$ ).

36 II, 437. En el ALEA II, 543 la misma forma, en plural, es más bien propia de la Andalucía oriental. Ver Alvar, Originalidad, $\$ 1.63$.

37 II, 459. También comentada por Alvar en Originalidad, $\$ 1.69$.

38 II, 308. Los nombres de pájaros en El Hierro no responden a la tónica general de las islas: en otras, capirote, segunda respuesta en $\mathrm{Hi} 3,4$ y 10 es la 'cogujada'. Abundan en El Hierro los casos de creación léxica, la confusión entre distintas aves, etc. Ver ALVAR, Originalidad, IS 1.38-1.41 y A. ESTÉveZ, I. MAS y P. SÁNCHEZ-PRIETO, «Los nombres de la alondra en Aragón y sus designaciones en otros ámbitos españoles», $A F A$, XXXIIXXXIII (1983), pp. 179-203.

39 I, 286. El portuguesismo es exclusivo de La Palma: borboleta (LP 1), barboleto (LP 3), barboleta (LP 30) port. borboleta 'id.', pero también está muy extendida paloma, forma andaluza, que El Hierro tampoco comparte.

40 I, 108. El Hierro conserva lavijero < clavijero, para 'clavija' en todos sus puntos y en Hi 4 también para 'clavijero'. Las demás islas se inclinan por chaveta, del port. id. «Peça de ferro, na extremidade de um eixo, para não deixar sair as rodas ou peça que segura uma cavilha». Ver ALVAR, Originalidad, \1.10.

41 I, 211. Tortullo es exclusivo de La Palma.

42 II, 548. Taramela, del port. id., «Peça de madeira que, girando em volta de um prego, cravado no batente de uma porta ou cancella, a fecha», se documenta en todas las islas salvo en La Palma y El Hierro. 
todas las islas - aunque hay ejemplos de mayor fidelidad a su origen ${ }^{43}$. El Hierro coincide, sobre todo, con La Palma pero, mientras que allí el portuguesismo se va quedando anticuado, aislado o se relega, el carácter uniforme del léxico herreño los suele conservar con un uso generalizado en plena vitalidad. Pero la regresión general de las palabras de origen portugués se ha iniciado también allí y los puntos más innovadores encauzan la penetración de castellanismos.

\section{GUANCHISMOS}

No son muchas las voces indígenas en El Hierro, lo que ha permitido a M. Alvar apuntar «la repulsa herreña hacia los términos prehispánicos»y establecer la posibilidad de que ésta se deba a «una muy precisa distribución del léxico en la isla» ${ }^{44}$. De hecho algunos guanchismos tienen aquí forma o significado distintos a los generales. P.e. jaira no se recoge y sí cabra mansa, y el 'cabrito' se llama chivo o chivito frente al baifo, baifito de otras islas, aunque El Hierro contesta unánime baifa para 'primala', allí donde las demás no concretan ${ }^{45}$. También las piedras del hogar se llaman tiniques, mientras que la forma generalizada es teníque ${ }^{46}$.

Otras palabras pertenecientes al léxico indígena, y ya muy estudiadas, son belete 'calostro', goro 'pocilga', majo 'abarcas' ${ }^{47}$. Goro se recoge sólo en $\mathrm{Hi}$ 1, como término anticuado, pero en $\mathrm{Hi} 3$ y 4 se llama gorona a la cerca que sirve para abrigar a las ovejas del viento ${ }^{48}$. Majo en $\mathrm{Hi} 1$ son 'correas de cuero para sujetarse los pies', en $\mathrm{Hi} 2$ como anticuado 'abarcas de los

43 M. Alvar, Originalidad, $\$ 1.54$, y el prólogo de J. Pérez Vidal a la Colección de voces y frases provinciales de Canarias de Sebastián de Lugo (La Laguna, 1946), especialmente las pp. 24-33.

44 Ibidem, IS $1.53,1.54$.

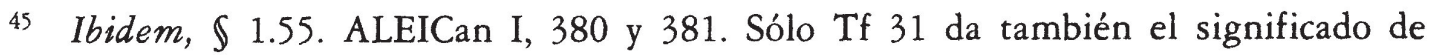
'primala' a baifa. J. Alvarez DelGado, "Ecero". Notas lingüísticas sobre El Hierro», II, $R H$, XII (1946), p. 157 afirma que no se emplean «las voces baifo "cabrito" y jaira "cabra", que conocen algunos como usadas en La Gomera y en Tenerife».

46 II, 574. En La Gomera son chinique (ver ALVAR, Originalidad, $\$ 1.83$ ) y sólo en Tf 31 tinique; en el resto, lo habitual es tenique. Para la explicación de las variantes, ver A. LloRente, «Comentario de algunos aspectos...», I Simposio, pp. 204-205.

47 II, 655, 556 y 538 respectivamente. En Hi 10 belete es también 'suero' (segunda respuesta).

48 Álvarez Delgado, "Ecero", p. 157, dice que sirve tanto para resguardar el ganado como los árboles, e incluye una fotografía de goronas. Quizá haya que relacionar con ellas la forma engoronado (m. 134, Hi 4), que significa 'brote a punto de reventar, pero sin poder hacerlo'. Ver M. Alvar, «Goro 'pocilga'», Estudios Canarios (Eds. del Excmo. Cabildo Insular de Gran Canaria: Las Palmas, 1968), I, pp. 111-114. 
pastores', lo mismo que en $\mathrm{Hi} 4$, donde el informante advierte que ahora se hacen con caucho de ruedas de camión ${ }^{49}$. También se registran en la isla guanchismos típicos de la flora y fauna autóctonas: tadaigo, con - $d$ - en Hierro y Gomera, frente al tasaigo general (I, 219), tabaiba ${ }^{50}$, tagasaste (I, 1. 303), mol(e) 'incienso' y perenquén ${ }^{\text {s1. }}$

Pero los mapas herreños dan otros posibles guanchismos: jere / jeren 'manija para hacer girar la piedra del molino de mano'; juaclo 'cueva' unas veces y otras 'choza de pastor', sin duda porque la primera hace las veces de la segunda ${ }^{52}$, y una serie de palabras relacionadas todas ellas con el color del ganado que parecen también exclusivas del Hierro: la cabra o vaca roja se llama cómboca en Hi 1 si es 'amarillenta' y cómbaca en Hi 10 s3; la blanca y negra se suele llamar pintada, pero en Hi 4, firanca si 'tira a negro' (I, 357) y en Hi 2 manajaisa es la 'oveja negra con la cabeza blanca' y pipana la 'blanca con la quijada canela' ${ }^{54}$.

Voces prehispánicas en lo más apegado a la vida primitiva: tíniques, majo, jere,, a las realidades paras las que el español no tenía significantes: tadaigo, tabaiba, tagasaste, perenquén, o a significados tan concretos que sólo el man-

49 Alvar, «Mago 'campesino'», en Estudios Canarios, I, pp. 115-120 y Alvarez DELGADO, 'Ecero", p. 164.

50 II, 474. Se llama tabaiba, choco de tabaiba al 'ahumador' de las colmenas, porque en todas las islas occidentales se quema tabaiba. Además de tabaiba (Hi 10,2), recibe el nombre de atabaibo en Hi 1 (I, lám. 303).

51 I, 223. No se ha podido establecer la filiación de mol ( $\mathrm{Hi} 2,3)$, mole (Hi 1, $10)$, voz exclusivamente herreña. Ver ALVAR, Originalidad, $\$ 1.23$, lo que hace pensar en un origen prehispánico. También se podría añadir sálamo 'bozal' (I, 299: Hi 4, 10), pues, aunque Guerra afirme que la voz «se ha oído alguna vez en Castilla», no se encuentra en ninguna recopilación de léxico peninsular.

52 I, 333. Juaclo es 'choza de pastor' en Hi 1 y 10 , aunque en Hi 2 se explica que se trata de una 'cueva para que las ovejas estén a la sombra'. En el mapa II, 789 Cueva, juaclo es en Hi 1, 2 'cueva pequeña', en Hi 10 'cueva para refugio del ganado' y en Hi 4 'cueva para encerrar el ganado en tiempo caluroso'. También Álvarez Delgado, "Ecero", p. 155, recoge juaclo, joaclo, xuaco como 'cueva'.

53 I, 356. En Hi 2 es cómboca 'la de color canelo oscuro con manchas'. Son voces no recogidas en los léxicos dialectales, pero Álvarez Delgado les dedicó un apartado titulado "Adjetivos de color herreños», en "Ecero", pp. 161-164, e intentó algunas aproximaciones a su primitivo significado. Su testimonio «Cómbaca o cóncava, canela clara con rayas rojas en cabeza y patas, o sea bermejo subido. Firanca y filanca, gris oscuro o revuelto de blanco y negro. Manajáis, manajáise o manajáiza, negra con lunar o mancha blanca en la cabeza. [...] Pípana, alunarada de varios colores, según otros blancaza con manchas oscuras en la quijada» coincide en lo esencial con los del ALEICan.

54 I, 358. Pípana debe pertenecer a la misma familia que puípana (m. 375 'lucero') 'animal con una mancha alrededor del ojo' y pípales, nombre que en $\mathrm{Hi} 10$ se da al ganado «calzado» (m. 376). 
tenimiento de las designaciones primitivas podía llenar: pípana, cómboca, manajaisa. Aunque no poseyéramos más que esto, su conservación ya nos indicaría la importancia tradicional del pastoreo en El Hierro 55 .

\section{CASTELLANISMOS}

Como hemos visto, el carácter castellano del Hierro es innegable pero, además, hay que destacar la conservación de voces antiguas que constituyen el «viejo fondo castellano vivo en la isla» ${ }^{56}$; M. Alvar puso de relieve algunos ejemplos como asta 'palo del pastor', panzón 'estómago del cerdo', condumio 'aliños para la matanza' ${ }^{57}$. El mapa 609 recoge para 'comida' ayanto y documenta también la existencia de ayantar 'comer'. Fuera del Hierro sólo ayento, voz arcaizante, es 'comida' en un punto de Tenerife y quedan restos con el sentido de 'cena' en Fuerteventura, La Palma y Gran Canaria. Ayantar / ayentar 'merendar' se encuentra un poco por todas las demás islas, pero aisladamente y en trance de desaparecer ${ }^{58}$. Quizá haya influido el portugués jantar en la conservación del castellano yantar, porque en Andalucía no hay ningún ejemplo de la voz y Figueiredo recoge también ajantarado «Semelhante a un jantar», por más que la $a$ - en verbos es común en Canarias.

También deben ser arcaísmos, más bien conservaciones locales, gorguero 'garganta de las personas' ${ }^{59}$, vianda 'presente de la matanza' ${ }^{60}$, beso 'labios

55 Para la importancia del ganado en la vida de los antiguos herreños ver Fr. J. de Abreu Galindo, Historia de la conquista de las siete islas de Canaria, ed. crítica con introducción, notas e índice por Alejandro Cioranescu (Santa Cruz de Tenerife: Goya eds., 1977), capítulos XVII y XVIII.

56 Ya a principios del XvII, Abreu Galindo decía que «El lenguaje que tienen es castellano, pues el suyo natural, ya lo han perdido, como todas las demás islas», Historia de la conquista..., p. 89. Ver ALVAR, Originalidad, \$1.69.

57 Alvar, Originalidad, IS 1.43, 1.69, 1.70. Lerner, Arcaísmos léxicos, s.v. señala que en América se sigue usando con diversos sentidos.

58 II, 609. En Hi 1, 2 es forma anticuada, pero no en los demás puntos. Su significado se ajusta al que el DRAE da como antiguo: "Comer al mediodía». Ayento 'comida' (Tf 31), 'cena' (Fv 3, LP 2), ayanto 'cena' (GC 2). Ayantar 'merendar' (GC 2, Go 3, Fv 30, Tf 50), ayentar 'id.' (Fv 3, Go 2, 40, Lz 3, 4, Tf 2, 6). Guerra para ayantar dice: «comida ligera de después del desayuno y antes del almuerzo» en los campos de Gran Canaria.

59 II, 491: Hi 1, 2, 10, en otras islas gualgüelo, garguero. En el ALEA (V, 1226) sólo encuentro garguero en Se 101. Garguero/gorgüero también en Cuba. Ver H. LOPEZ MORALES, «Relaciones léxico-semánticas en el ámbito lingüístico canario-cubano», en I Simposio, p. 314.

60 II, 446: Hi 1 y 10. 
de los animales' ${ }^{61}$, nacido 'ántrax' ${ }^{62}$, y logrero 'usurero' ${ }^{63}$. Hay casos en los que la palabra puede documentarse en el ALEA: p.e. tután(o) 'tuétano' ${ }^{64}$, cuero 'piel' "5, servidumbre 'entrada de un campo' 66, jabardo 'hatajo' ${ }^{67}$ y ajotar 'azuzar' ${ }^{68}$.

61 II, 490: Hi 2, 3, 4. Se encuentra en La Palma y Fuerteventura. En Lanzarote, Gran Canaria y Tenerife es arcaísmo. Hi 1 dice belfos.

62 II, 526. Se registra con el sentido de «divieso, nacencia» en el DRAE. También en Puerto Rico, el Caribe en general y otros países de América, según M. ÁlvareZ NAZARIo, «Relaciones histórico-dialectales entre Puerto Rico y Canarias», en I Simposio, p. 304. Para Cuba, ver también H. LOPEZ MORALES, art. cit., p. 320; quizá pasó a allí desde Canarias.

63 II, 675. Es exclusivamente herreña. AlvaR, Originalidad, $\mathbb{S} 1.90$, señala que la voz parece ser un lusismo por su localización occidental en Andalucía. En port. es antigua y significa «O mesmo que usurário». Pero es que también en lo antiguo logrero se empleó en castellano por 'usurero': J. COROMINAS y J. A. PASCUAL, en su DCECH, s.v. documentan la forma en Berceo, pero también en El Conde Lucanor, Don Juan Manuel incluye un Exemplo, el XIII, titulado «Del miraglo que fizo sancto Domingo quando predicó sobre el logrero» (ed. de J. M. Blecua, Madrid: Clásicos Castalia, 1971). (Dato que me indicó amablemente J. M. Fradejas). Similar resulta el caso de gago 'tartamudo' (II, 524), que Corominas y Pascual dan como antiguo, canario, salmantino y americano. El hecho de que no se documente en el ALEA y de que tenga igual forma y significado en portugués puede hacer pensar o bien que el port. gago influyó en su conservación canaria o que se trate de un lusismo en las islas. ÁlvarEz NAZARIO, art. cit., p. 304 lo registra en Puerto Rico y también H. LÓPEZ MORAles en Cuba, art. cit., p. 320. ¿Arcaísmo en Canarias y América o lusismo canario en América? Lerner, Arcaísmos léxicos, s.v.

64 II, 493. En las demás islas tútano es la forma dominante, aunque ya alterna con tuétano, pero El Hierro presenta apócope muy extendida: el tutan (Hi 4, 10), el tuta (Hi 1) y los puntos marineros el tuétan (Hi 2,3), alternando en Valverde (Hi 2) con tuétano. Tútano es frecuente en Andalucía (ALEA V, 1231) y Zamora ViCENTE, Dialectología española, p. 385, lo señala como típico del español de América, especificando «arcaísmo, ya en La Celestina». Coincide, además, con la forma portuguesa tutano.

65 II, 477 ad. Cuero 'piel' en Gc 2, 20, 30, Tf 40 y en Hi 2 y 3 . En Hi 3 es vulgar o se dice de los animales. En Andalucía hay restos en Jaén (J 101 cv.) y Almería (Al 300 y 405: ALEA V, 1176). También es arcaísmo en América, LeRner, op. cit., s.v..

66 I, 12. 'Entrada de un campo' en casi todas las islas es serventía, que el DRAE da como propio de Asturias, Canarias, Cuba y Méjico, y que quizá habría que ver como portuguesismo canario o como occidentalismo apoyado por un segundo sentido del port. serventia «Servidão. Passagem. Passadiço», pero en El Hierro, La Palma y Tenerife alterna con servidumbre. En el ALEA IV, 874 sólo un punto de Málaga (401) llama al 'sendero' cegiumbre, verea.

67 I, 34. Jabardo en Hi 2, 10, jabardito en Hi 1. Como 'hatajo' es término exclusivo del Hierro. El DRAE lo define como «enjambre pequeño producido en una colmena como segunda cría del año», de jarbar< lat. e x s e p à r a r e 'separar', sentido para el que la isla emplea unánime enjambre (II, 472: Tf 4 y 40 dan, junto a enjambre, jabardo y abardo). Esto podría hacer pensar en un cambio herreño 'pequeño enjambre' $>$ 'hatajo', si no fuera que el ALEA II, 444 documenta jabardillo para 'hatajo' en J 204, 205, Co 403 y Ca 301.

68 II, 414. General en El Hierro y Gomera; también en LP 2, 3 y Gc 3, 30. Según LERNER, op. cit., s.v., se oye en Asturias y se conserva en América como 'azuzar, instigar'. 
Castellanismos - con vida en Andalucía-, pero esta vez exclusivos del Hierro en el conjunto del Archipiélago, son jornalero 'trabajador eventual' ${ }^{69}$, lavijero 'clavija' ${ }^{70}$, mosca de bestia 'tábano' ${ }^{71}$, requesones 'requesón' ${ }^{72}$, talega 'zurrón' ${ }^{73}$, nacimiento 'manantial' ${ }^{74}$, cortahierro 'cortafrío' ${ }^{75}$. Llama la atención encontrar en El Hierro amapola, marrubio, almorzada o sanantón 'mariquita' bajo su apariencia más castellana frente a mabapola / mapola, malrubio, ambuesa / ambosada, sarantontón, etc. de otras islas ${ }^{76}$. En ocasiones se dan adaptaciones herreñas del fondo castellano: p.e. postrero es 'fruto tardío' en Gomera y El Hierro, pero también 'hijo nacido tardíamente' 77 , estofar es 'recentar' y estofarse 'crecer el pan' ${ }^{78}$, empanar 'sembrar a voleo' 79, burgonero 'barredero de horno' ${ }^{80}$ y espichar 'plantar hortalizas' ${ }^{81}$.

Pero junto a la conservación, El Hierro manifiesta una sorprendente capacidad de creación léxica. Por otra parte, su tendencia a la uniformidad suele generalizar lo que quizá, en otra isla, no habría pasado de ser un testimonio casi aislado. Vimos los casos de rueda 'gajo de la naranja' y cabra

Pero el ALEA documenta su conservación en Andalucía, donde, sin embargo, no es general (H 102, 200, 202, 204, 300, 400, 500 y Co 102 y 202) y suele deformarse.

69 I, 33. Muy frecuente en Andalucía (ALEA IV, 928). Es la respuesta de Hi 2 y 10; en Hi 1, obrero y en $\mathrm{Hi} 4$, hombre.

70 Ver nota 41.

71 I, 287: Hi 1, 2, 3, 10. En el ALEA II, 379: H 300, 302, Se 404 y 600.

72 Ver nota 37.

73 I, 332. en Andalucía resulta muy habitual, junto a talego, taleguilla, etc. ALEA II, 497: Se 306, 309, 406, 601, Ma 407, Co 604, 606, Al 501.

74 II, 781. En Hi 4, 3, 10, aunque las demás islas prefieren naciente, quizá del port. nascente «Ponto, onde nasce ou começa uma corrente de água; fonte; manancial». Nacimiento en andaluz (ALEA IV, 894).

75 II, 643. En Andalucía se documenta en H 504, Ca 500 y 602 (ALEA IV, 977).

76 Ver mapas I, 214, 215; II, 505; I, 293 respectivamente.

77 La primera acepción en I, 249. Ver Alvar, Originalidad, $\mathbb{1}$ 1.26; la segunda en II, 633: postrero (Hi 2, 4) y postrerito (Hi 1, 3, 10). También se documenta en Go 2, $\mathrm{Lz} 1,20$. No tienen paralelos andaluces.

78 I, 178 y I, 181. Ver AlVAR, Originalidad, $\$ 1.19$.

79 I, 36, 'sembrar a voleo' (Hi 1, 4, 10), 'sembrar patatas' en $\mathrm{Hi}$ 2, porque allí se ha introducido ya sembrar. No lo registra el ALEA, pero podría ajustarse a la tercera acepción del DRAE «Sembrar de trigo las tierras».

80 I, 196, cuando lo habitual en las islas y Andalucía es que designe al 'tirabrasas', que en El Hierro se llama rastrillo (m. 197). Ver AlvaR, Originalidad, $\mathbb{S} 1.20$. El Hierro participa de alguna adaptación general en las islas, como la de sereno > 'rocío'.

81 I, 37. En Hi 1; en Hi 2 es 'sembrar maíz'. Quizá haga referencia a la forma de plantar, porque su significado académico es el de "punzar con una cosa aguda». No se documenta en el ALEA. 
mansa 'cabra doméstica', pero también son representativos barbanes 'mamellas' ${ }^{82}$, araña mamona 'tarántula' ${ }^{83}$, saltones 'renacuajo' ${ }^{84}$, tripa (del) cabo 'tripa cular' ${ }^{85}$, pelo vaca / color de vaca 'pelirrojo' ${ }^{86}$, hierro 'cencerra' ${ }^{87}$, bailarines 'burbuja' ${ }^{88}$, estrella (de)l agua 'lucero de la tarde', andoriña de ratón/ratona 'murciélago' ${ }^{89}$, clavijo 'aguijón' ${ }^{90}$, tirito 'espeluzno de frío' ${ }^{91}$, desponer 'huevo sin cáscara' ${ }^{92}$, cielo encofrado 'cielo emborregado' ${ }^{93}$, arado / arado con gañán 'osa mayor' ${ }^{94}$.

82 II, 387, frente al general mamellas. Ver ALVAR, Originalidad, $\$ 1.57$.

83 I, 301. La motivación es evidente. Sólo Hi 2 añade, como segunda respuesta, araña negra.

84 I, 304. Sólo se obtuvo la extraña respuesta en Hi 2 y 10. Como comenta ALVAR, en Originalidad, $\ 1.37$, el saltar no resulta característico del renacuajo. ¿Tendrá que ver con el port. saltão que A. BUARQue, op. cit., define como «mosquito, antes de completar a sua metamorfose»? gorda, etc.

85 II, 453. Denominación exclusivamente herreña frente a tripa (de)l culo, tripa

86 II, 483. Pelo vaca (Hi 2, 1, 3), color de vaca (Hi 10, 4). En Andalucía sólo H 502 da la primera respuesta. Ver el comentario de ALVAR, Originalidad, $\$ 1.73$.

87 I, 337: Hi 1, 4, 10, sin duda por el material del que se hacen.

88 II, 776. Es respuesta, en plural, de $\mathrm{Hi} 1,2,10$. En Hi 10 gorgoritas y en Hi 3 borborita, de acuerdo con otras islas. Sorprendentemente Hi 4 responde fraire.

89 I, 317. Las denominaciones del murciélago son harto extrañas en El Hierro. ALVAR dedicó un comentario en Originalidad, $\$ 1.42$, a ratona ( $\mathrm{Hi} 4,3$ ), andoriña $(\mathrm{Hi} 1)$, andoriña de ratón, explicando estas últimas a través de posibles creencias populares. También $\mathbf{M}$. R. Simoni-Aurembou, «Nombres de algunas bestezuelas en Andalucía y Canarias», I Simposio, se ocupa de ellas y las explica por un temor supersticioso hacia el animal (p. 131). De cualquier forma, El Hierro, como vimos, es anormalmente inconcreto en los nombres de aves, que suele trastocar. En el caso de andoriña de ratón quizá simplemente haya que ver la equivalencia ratón-murciélago, más algo que expresara su condición de volador, a no ser que refleje efectivamente alguna tradición perdida.

90 II, 476. Ver AlVAR, Originalidad, $\$ 1.72$.

91 II, 514. Teritar 'tiritar' en Hi 1 y escalafrio en Valverde (Hi 2). Tirito (Hi 4, 3, 10) es un postverbal de tiritar, exclusivo de la isla.

92 II, 413. (D)esponer (Hi 1, 2, 10), descompuesto (Hi 4), se despuso (Hi 3). Ver ALVAR, Originalidad, $\$ 1.61$.

93 II, 751 . General en la isla que utiliza insistentemente el lenguaje de la construcción, pues en Hi 10 es también enladrillado, en cofre y en Hi 4 empedrado. Ver Alvar, Originalidad, $\$ 1.94$.

94 II, 754. En las islas es bastante frecuente arado ( $\mathrm{Hi} \mathrm{1,2)} \mathrm{y} \mathrm{carro} \mathrm{(Hi} \mathrm{4).} \mathrm{Este}$ último, y también el carro y las mulas, en Andalucía (ALEA IV, 838). Quizá la no utilización del carro haya llevado a crear una forma paralela: arado con gañán (Hi 10), arado gañán (Hi 1). 


\section{VOCES MARINERAS}

Es El Hierro, como todas las islas, tierra abonada para las voces marineras. Al no estudiar el tomo III, no son muchas las que se pueden allegar: sí resulta curioso que se diga de las cabras y vacas en celo que están con marea o con buena marea ${ }^{95}$. Además de los generales empatar 'añadir, alargar' ${ }^{96}$, remo 'columpio' ${ }^{97}$ y corsa 'narria' ${ }^{98}$, quizá gaveta se podría explicar como una extensión de la acepción marinera del término. Gaveta en El Hierro es un objeto de variada utilidad: 'artesa', 'dornajo' o 'vasija para fregar', según las localidades ${ }^{99}$. Estos significados no concuerdan con los de gaveta en español, ni en portugués, pero sí pueden ser una adaptación de los secundarios que el DRAE recoge con la marca de marinerismo «Tina pequeña de madera para servir la comida. Balde pequeño para el vino». Asimismo, se podría ver influencia del lenguaje del mar en arrife, si lo consideramos portuguesismo procedente de arrecife ${ }^{100}$.

\section{VOCES AMERICANAS}

Muchas veces se ha hecho hincapié en el papel de las Canarias como puente entre España y América. Las islas dieron puerto, productos y hombres a las embarcaciones que cruzaban el Atlántico ${ }^{101}$. El Hierro fue desde antiguo tierra de emigrantes. Sus hombres marchaban a Tenerife y América: Vene-

95 I, 345 '(vaca) torionda', pero también en los mapas 364, 397 y II, 443. Tf 20: tiene marea, $\mathrm{Lz}$ 4, 3, 10, 20 en marea.

96 I, 111. Muy extendido.

97 II, 698. En El Hierro, Gran Canaria, Fuerteventura y Lanzarote.

98 Si se acepta la tesis de J. FernándeZ Sevilla, en «Andalucía y Canarias...», pp. 85-87, quien lo relaciona con el lat. c u r s u s.

99 I, 176: 'artesa' en Hi 1, 10; II, 450 'dornajo' en Hi 3 (está hecho de un tronco vaciado); II, 600 'vasija para fregar' en Hi 4. Es término antiguo (era de madera), hoy se prefiere lebrillo. Todas las gavetas herreñas tienen en común el ser de madera y vienen a coincidir con los testimonios que Max STEFFEN, «Lexicología canaria I. 1. Til. 2. Gaveta y dornajo. 3. Gilbarbera. 4. Embelesar», Revista de Historia, XI (1945), pp. 142-146, recoge de otras islas (Gomera, La Palma y Gran Canaria). Parece que ya en Mateo Alemán se encuentra con este sentido. Ver COROMINAS y PASCUAL, DCECH, s.v. De los usos que Lerner recoge en América, op. cit., s.v., sólo el argentino «'dos recipientes unidos en los que se coloca la yerba y el azúcar para el mate'» se podrían relacionar con la gaveta canaria.

100 Ver nota 32.

101 Cfr. los trabajos citados de M. Álvarez Nazario y H. Lopez Morales. También F. MORALES PADRÓN, El comercio canario-americano (Sevilla: Escuela de Estudios Hispanoamericanos, 1955) y M. Álvarez Nazario, La buella canaria en Puerto Rico (San Juan: Instituto de Cultura Puertorriqueña, 1976). 
zuela, México, Cuba, Puerto Rico eran algunos de los destinos habituales. La pérdida de las colonias supuso la vuelta de algunos indianos enriquecidos a la humilde isla del Hierro, pero la emigración continuó y todavía hoy va preferentemente dirigida hacia Venezuela ${ }^{102}$. Estas circunstancias explican que el español de las islas presente voces americanas a veces desconocidas en la Península. No existen en El Hierro americanismos exclusivos, todos reaparecen especialmente en La Palma y Tenerife: p.e. guataca, que el DRAE da como voz cubana «Azada corta que se usa para limpiar de hierba las tierras» ${ }^{103}$, es en El Hierro 'azada' y 'raedera'; en Hi 1 la segunda respuesta para 'gemelos' fue jimaga y, además, el informante tenía conciencia de que «la traen de Cuba», como confirman los diccionarios ${ }^{104}$. Llaman alegantín en Hi 2 y 3 al 'charlatán', palabra americana, igual que el alegador de otras islas ${ }^{105}$.

De todas formas, es difícil a veces señalar si un término que, en definitiva, se remonta al castellano es, en determinada acepción, americanismo en Canarias o isleñismo en América. El Atlas Lingüístico de Hispanoamérica resultaría fundamental para estudiar con seriedad la huella de España en América ${ }^{106}$.

\section{PUNTOS DE ENCUESTA: CONSERVACIÓN E INNOVACIÓN}

Es fácil observar en El Hierro la coincidencia de respuestas de $\mathrm{Hi} \mathrm{1,} 10$ $\mathrm{y}$, a veces, $\mathrm{Hi} 4$. Los puntos marineros ( $\mathrm{Hi} 2$ y 3) son los que pueden disentir de la forma general y, cuando lo hacen, aceptan palabras propias

102 D. V. DARías y PADRÓN, Noticias generales históricas sobre la Isla del Hierro (Tenerife, 1980), pp. 199-200 y M. Alvar, Niveles socio-culturales en el babla de Las Palmas de Gran Canaria (Las Palmas: Eds. del Excmo. Cabildo Insular, 1972), pp. 264165, mapa 1 dedicado a la emigración.

103 Parece ser un tipo de azada en forma de media luna. En Hi 1 y 10 también se emplea azada, pero es término antiguo. En Hi 4 conocen también sacho. Fuera del Hierro, guataca se encuenta en La Palma, Gomera, Tenerife y Lanzarote. En todas es bastante frecuente para 'raedera' (II, 633). Según el Diccionario de cubanismos más usuales de J. SÁNCHEZ-BOUdY (tesis en publicación), guataca se llama a una «Especie de azada con que se corta la hierba».

104 II, 652. Imagua es general en Gomera, y también se documenta en LP 1, 3, 10. SÁNCHEZ-BOUdY da para Cuba jimagua, voz que también recoge LópEZ MorALES.

105 II, 678. En GUERRA, op. cit., alegantina «Mujer murmuradora, chismosa», igual que A. Millares Cubas, Cómo hablan los canarios, Las Palmas [¿1932?], pp. 6-7.

106 Ya H. LÓPEZ MORALES señaló en 1978 la necesidad de su realización, art. cit., p. 317. Ver M. Alvar y A. Quilis, Atlas Lingüístico de Hispanoamérica. Cuestionario (Madrid: Instituto de Cooperación Iberoamericana, 1984). 
de otras islas o de la norma. Los puntos interiores son, sin duda, más conservadores y Valverde, a pesar de participar del léxico de otras islas, suele conservar la voz herreña junto a la innovación: p.e. (II, 533) 'corpiño' es cuerpillo en Hi 3 y cuerpiño en Hi 2, pero Hi 1, 4 y 10 lo llaman justillo; (II, 583) el castellanismo pabilo se conserva en $\mathrm{Hi} 1,2,4$ y 10, en $\mathrm{Hi} 3$ y 2 es mecha; (II, 616) 'mendigo' es limosnero, sólo Hi 2 disiente con pobre; (II, 712) margullir vale para 'chapuzar' en todos los puntos, pero el informante marinero de Valverde conoce también zambullir (Hi 2).

La isla resulta conservadora en los nombres de los meses: los puntos interiores (1, 4 y 10) siempre coinciden en los nombres tradicionales, alusiones a la fiesta religiosa más señalada del mes: Hi 2 y 3 darán, en cambio, el nombre «oficial», aunque en Valverde el nombre del interior suele ser segunda respuesta. 'Junio', p.e., es San Juan en los puntos interiores, Junio/San Juan en Valverde y Junio en Hi 3. El mismo esquema se repite para 'julio', 'noviembre' y 'diciembre', Santiago, San Andrés y Navidad en el interior ${ }^{107}$.

En resumen, Sabinosa (Hi 10), Frontera (Hi 1) y Taibique (Hi 4) resultan conservadores y bastante uniformes; Valverde ( $\mathrm{Hi} 2$ ) suele participar de las soluciones de los puntos anteriores, pero su vida hacia el mar y su condición de núcleo hacen que esté más abierto a lo nuevo, y, finalmente, la Restinga ( $\mathrm{Hi} 3$ ) parece depender sólo del mar, por lo que se aparta a veces de los puntos interiores y, como es lógico, coincide en ocasiones con Valverde. La innovación, la incorporación a la norma canaria, llega a la isla por el mar ${ }^{108}$.

\section{CONCLUSIONES}

Las principales características del léxico herreño se derivan de las condiciones de la isla: isla marginal, sin grandes puertos en las rutas de navegación. Su conservadurismo se debe a todas estas causas y al hecho de tener una población no renovada que emigra con frecuencia. Es poca la influencia lingüística de las grandes islas pero, en cualquier caso y como es lógico por su historia y su geografía, se vincula más con Tenerife, La Palma y la Go-

107 Quizá también influya el tipo de vida: pueblos agrícolas y ganaderos frente a pueblos marineros. (Junio (m. 727), julio (m. 729), noviembre (m. 737), diciembre (m. 739). Las denominaciones San Juan, Santiago, etc., suelen darse como antiguas en puntos del interior de otras islas.

108 Alvar, «Geografía y sociología lingüísticas en el español insular», Estudios Canarios, I, pp. 27-29 especialmente, donde se muestra en desacuerdo con quienes afirman sin más que la lengua de los marineros es arcaizante. 
mera, las islas occidentales. La creación que marca en muchas ocasiones el léxico del Hierro es consecuencia de una sociedad cerrada, que vive hacia dentro, con una economía casi familiar. Estas mismas condiciones, apoyadas en el pequeño tamaño de la isla y en la ausencia de importantes núcleos urbanos que irradien novedades lingüísticas, explican la nivelación que motiva la clara uniformidad del léxico. Sería útil un estudio detallado sobre los primeros pobladores del Hierro después de la conquista, ya que el elemento portugués, como en La Palma y Tenerife, parece importante, especialmente en el ámbito agrícola. De cualquier forma, se debe señalar la presencia de voces prehispánicas, aunque muy restringidas al campo de la ganadería, la flora y la fauna.

En comparación con el léxico de las grandes islas - y a pesar de su clara relación con Andalucía-, muchas de sus voces parecen castellanas y antiguas. Quizá esto se deba a una especial confluencia de normas portuguesaleonesa-castellana y andaluza y al posterior aislamiento que impediría su incorporación a las tendencias de la «norma» canaria.

\author{
Pilar García Mouton \\ Instituto de Filología \\ C.S.I.C., Madrid
}

A partir de los mapas lingüísticos del Atlas Lingüístico y Etnográfico de las Islas Canarias (ALEICan), se intenta caracterizar el léxico de la conservadora isla del Hierro, clasificándolo en portuguesismos, guanchismos, castellanismos y voces marineras.

Through the use of the ALEICan (Atlas Lingüístico y Etnográfico de las Islas Canarias), we try to characterize the lexicon of the conservative island of El Hierro, classifying the origine of the words as portuguese, guanche, castillian, etc. 